\title{
Data-Driven Control Design for Neuroprotheses: A Virtual Reference Feedback Tuning (VRFT) Approach
}

\author{
Fabio Previdi, Thomas Schauer, Sergio M. Savaresi, and Ken J. Hunt
}

\begin{abstract}
This paper deals with design of feedback controllers for knee joint movement of paraplegics using functional electrical stimulation (FES) of the paralyzed quadriceps muscle group. The controller design approach, virtual reference feedback tuning (VRFT), is directly based on open loop measured data and fits the controller in such a way that the closed-loop meets a model reference objective. The use of this strategy, avoiding the modeling step, significantly reduces the time required for controller design and considerably simplifies the rehabilitation protocols. Linear and nonlinear controllers have been designed and experimentally tested, preliminarily on a healthy subject and finally on a paraplegic patient. Linear controller is effective when applied on small range of knee joint angle. The design of a nonlinear controller allows better performances. It is also shown that the control design is effective in tracking assigned knee angle trajectories and rejecting disturbances.
\end{abstract}

Index Terms-Direct controller design, functional electrical stimulation (FES), neuroprostheses, virtual reference feedback tuning (VRFT).

\section{INTRODUCTION}

$\mathbf{T}$ HE aim of this work is the design of a controller of neuroprostheses for paraplegic patients suffering from spinal cord injury. In a paraplegic subject the muscles of the lower limbs are intact and capable of effective contraction, but there is the fundamental lack of stimulation from the brain through the injured spinal cord. Functional electrical stimulation (FES) can provide the missing electrical stimuli to induce muscle contraction and the corresponding joint movement. In the case of this work, by stimulating the quadriceps muscle group a movement of the knee joint can be obtained. So, by measuring the knee joint angle it is possible to design a feedback controller to decide the electrical stimulation necessary to achieve predetermined movements of the knee. Such a controller can be useful in more complex motor functions such as cycling, standing up, sitting down, or stepping [1]-[3].

In literature, the available strategies to obtain neuroprostheses can be grouped into two major categories: patient-centered ap-

Manuscript received October 25, 2002; revised May 26, 2003. Manuscript received in final form October 8, 2003. Recommended by Associate Editor S. Kim. This work was supported in part by the U.K. Engineering and Physical Sciences Research Council through grant GR/M47256/01.

F. Previdi is with the Dipartimento di Ingegneria Gestionale e dell'Informazione, Universitá di Bergamo, Dalmine (BG) 24044, Italy (e-mail: previdi@unibg.it).

T. Schauer is with the Max Planck Institute for Dynamics of Complex Technical Systems, Magdeburg D-39106, Germany (e-mail: schauer@mpi-magdeburg.mpg.de).

S. M. Savaresi is with the Dipartimento di Elettronica e Informazione, Politecnico di Milano Piazza, Milano 32-20133, Italy (e-mail: savaresi@elet.polimi.it).

K. J. Hunt is with the Centre for Rehabilitation Engineering, Department of Mechanical Engineering, University of Glasgow James Watt, Glasgow G12 8QQ, Scotland, U.K. (e-mail kenh@mech.gla.ac.uk).

Digital Object Identifier 10.1109/TCST.2003.821967 proaches and controller-centered approaches. In the first case, the paraplegic patient is able to influence the stimulation of the lower limbs. In the second case, the patient limbs are stimulated to track a predetermined trajectory.

Two examples of the first method are CHREMLS [4] and PDMR [5], both aiming to the control of standing up in paraplegics. In CHREMLS, the stimulation depends on the upper body effort, i.e., on body posture and recorded hand reactions, aiming to minimize arm effort during standing up. The stimulation of the leg muscles is adapted in order to minimize handle reaction forces. This is obtained by using a map of the relation between handle forces and leg joint movements, i.e., an inverse model of the plant. In PDMR, no measurement of the hand reaction is needed and the stimulation pattern is directly computed by means of a dynamic inverse model of the plant, in order to maintain the movement initiated by the patient. Strictly speaking, both CHREMLS and PDMR are not closed loop approaches. In fact, the controlled variable is measured and fed back to an inverse model of the plant dynamics. So, this control scheme will suffer all the major drawbacks of open loop control design.

Two examples of the second method can be found in [6] and [3]. In the first paper, a nonlinear regulator is proposed for the control of the knee joint movement. In particular, a gain scheduling controller is designed by interpolation of a set local linear quadratic (LQ) regulators. Each LQ controller is designed on the basis of a local linear model obtained by linearization of a nonlinear model estimated from input-output (I/O) data. In [3] pole placement is used to obtain a linear controller for the knee joint angle. The controller is based on the highest gain linear autoregressive exogenous (ARX) model chosen from a set of linear models of the plant directly estimated from I/O data or obtained by linearization of a nonlinear model as in the previous case.

Following a "controlled-centered" approach, the first step for the design of a neuroprosthesis is usually a modeling stage: an I/O dynamical system describing the relationship between the input FES pattern and the knee joint position is obtained (see for instance [6]-[14]). In most cases, black-box models are estimated from an I/O sequence collected on the patient. The second step is the design and test of a controller based on the estimated model (see [6], [8], [12]-[16]). So, the procedure to design a "controller-centered" neuroprosthesis could be time consuming.

In this paper, the application of virtual reference feedback tuning (VRFT) strategy to the challenging problem of controlling joint positions by means of electrical stimulation of muscles will be presented and results obtained in experimental sessions will be discussed.

The VRFT method gives a solution to the problem of designing a controller for a system, whose I/O behavior is un- 
TABLE I

ANTHROPOMETRIC DATA: $m_{B}$ - BODY WEIGHT, $h_{B}$ - HEIGHT, $m$ - MASS OF THE SHANK-FoOT COMPLEX $\left(m=0.061 m_{B}\right), l_{s e q}$ - SHANK-FoOT SEGMENT LENGTH $\left(l_{\text {se } g}=0.28 h_{B}\right), d$ - DistanCE OF CENTRE OF MASS FROM THE PROX. END $\left(d=0.61 l_{s e q}\right), J_{K}$ MOMENT OF INERTIA OF SHANK AND FOOT $\left(J_{k}=0.0020 m_{B} h_{B}^{2}\right)$

\begin{tabular}{lcc}
\hline Subject & Healthy & Paraplegic \\
\hline Age & 27 & 58 \\
\hline$m_{B}[K g]$ & 85 & 84 \\
\hline$h_{B}[m]$ & 1.76 & 1.62 \\
\hline$m[K g]$ & 5.18 & 5.12 \\
\hline$l_{\text {seg }}[\mathrm{m}]$ & 0.49 & 0.45 \\
\hline$d[\mathrm{~m}]$ & 0.30 & 0.28 \\
\hline$J_{K}\left[\mathrm{~kg} \cdot \mathrm{m}^{2}\right]$ & 0.53 & 0.44 \\
\hline
\end{tabular}

known, on the basis of a single set of I/O data without resorting to the identification of a model of the system.

The idea on which VRFT is based was originally proposed in [17]-[21] and developed in [22], [23] as a complete and ready to use method for data-driven control design in a noisy environment. The aim of the VRFT approach is not the design of the "best" possible controller, but the design of a controller with good tracking and robustness properties, based on a reduced set of data and so tunable in very short time. The VRFT method have given promising results also in different fields of application [24] and in other FES control problems [1].

Preliminary results based on simulations and a first experiment on a healthy subject were presented in [25]. In this paper, experimental results on healthy subjects and paraplegics obtained using linear and nonlinear controllers designed with VRFT will be discussed. The experimental results presented in this paper have been obtained in the laboratory of the Centre for Rehabilitation Engineering of the University of Glasgow.

The structure of the paper is the following: in Section II the experimental setup is described. This experimental protocol is the fruit of a long and consolidated work on such control problems and it can now be considered as a standard in FES control applications. Moreover, the knee-joint control problem is widely used as a benchmark for studying FES control strategies [6], [8], [13], [15]. In Section III the control strategy adopted in the experiments is described. Then, in Section IV the experimental results obtained on healthy and paraplegic subjects are presented. Then a nonlinear modification of the control algorithm is proposed and tested on a paraplegic patient.

\section{EXPERIMENTAL SETUP AND PROTOCOL}

The experiments have been carried out on two subjects: a 27-years-old healthy male and a 58-years-old paraplegic subject, four years past from an injury at T10 level, i.e., an injury at the 10th thoracic vertebra. The anthropometric data of the two subjects are reported in Table I. During each session the subject is sitting on a bench with the unloaded shank free to swing (cf. Figs. 1 and 2). The knee angle $\Theta$ in degrees is measured in realtime by an ultrasound-based 3-D-motion analysis system [26]. The angle is directly calculated from the measured positions of three markers placed at the ankle, knee, and close to the hip, approximately aligned with the femoral bone. This sensor system is connected to a laptop computer by a proprietary interface.

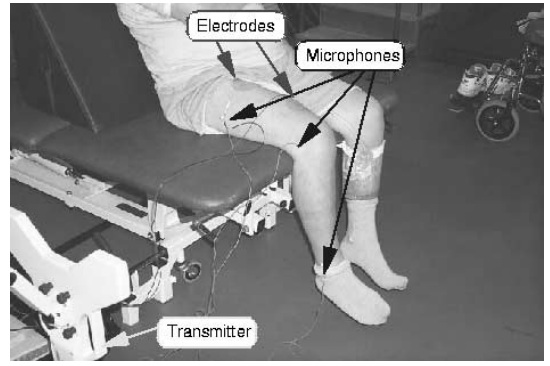

Fig. 1. Position of the subject during the experiments. The three markers (microphones) of the ultrasound motion analysis system are visible at the knee, at the ankle and close to the hip, aligned with the femoral bone. The electrodes are visible just above the hip and knee markers. On the left side a part of the ultrasound transmitter unit can be seen.

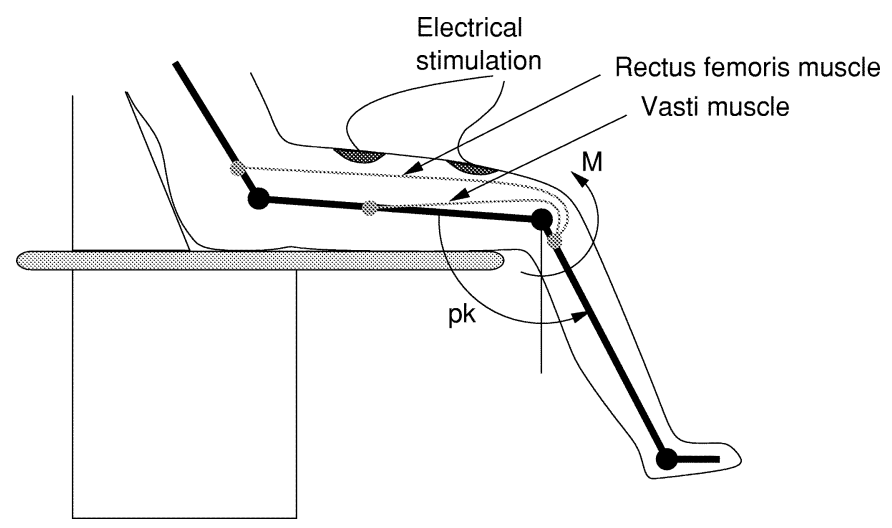

Fig. 2. Definition of the knee joint angle $\Theta$. The active knee moment $M_{a c t}$ is induced by electrical stimulation of the quadriceps muscle group (rectus femoris and vasti muscle).

First of all, the knee angle is normalized so that it varies from $y=0$ at rest position without any stimulation to $y=1$ at maximum knee extension when the muscle is fully contracted. Rest position and maximum knee extension are in general not equal to $\Theta=90^{\circ}$ and $\Theta=180^{\circ}$, respectively. In particular, in a paraplegic these values could be considerably reduced (about $120^{\circ}-150^{\circ}$ ), as a consequence of joint contractures or muscle retraction.

The data are collected with a sampling time $T_{s}=0.05 \mathrm{~s}$. The quadriceps muscle group is stimulated in a nonselective way using surface electrodes which deliver the electrical pulses generated by a portable stimulator connected to the laptop computer. The frequency is $f=20 \mathrm{~Hz}$ and the electrical charge delivered to the muscle is modulated by changing the pulsewidth. So, the actual control variable is the modulated pulsewidth measured in $\mu \mathrm{s}$. All the implementation is done in MATLAB/SIMULINK (MATLAB/SIMULINK is a registered trademark of the The Math Works, Natick, MA) using Real Time Toolbox (Real Time Toolbox is a registered trademark of Humusoft, Prague, Czech Republic) [27].

Each experimental session is made of three main steps.

At the beginning, a preliminary test is made in order to choose the stimulation current (usually about $I=40 \ldots 80 \mathrm{~mA}$ ). Starting with a low current, the pulse width is ramped up in steps of about $25 \mu$ s from $50 \mu$ s to about 400-500 $\mu$ s and the angle is measured (see Fig. 3). Then the current is incremented by $10 \mathrm{~mA}$ and the stimulation pattern is repeated. This process 

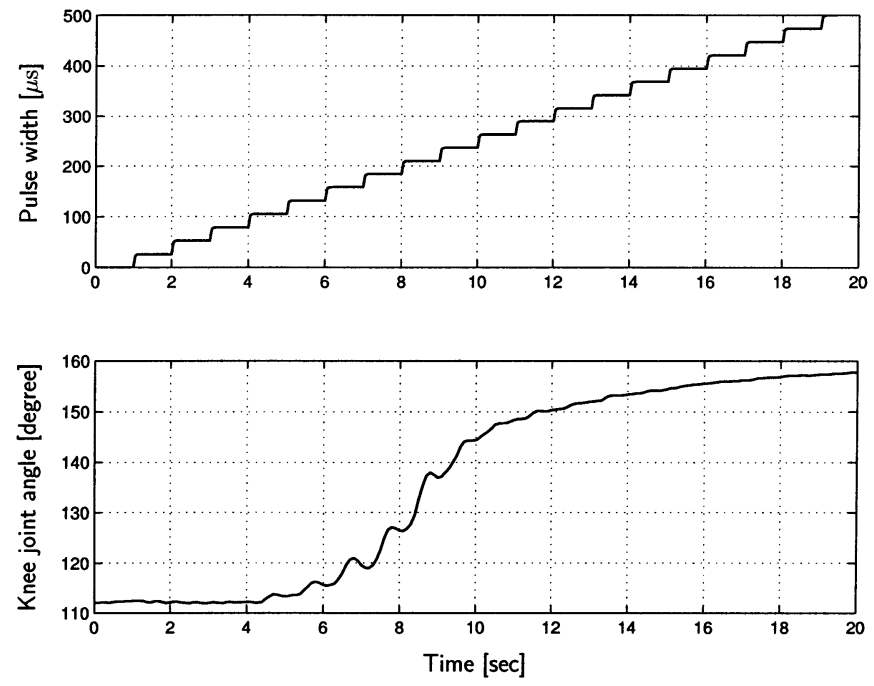

Fig. 3. Results of the preliminary test with $I=80 \mathrm{~mA}$ on a paraplegic The upper part of the plot represents the pulsewidth of the electrical stimulation (input). The lower part is the knee joint angle (output).

continues until the knee is close to full extension at high pulsewidths. The current is fixed at this level for the remainder of the experiment. In the experiments presented in this paper, $I=60 \mathrm{~mA}$ and $I=80 \mathrm{~mA}$ are the current values used for the healthy subject and the paraplegic subject, respectively. The input (pulsewidth) is desaturated and normalized to the range $0-1$. This is done using the result from the previous preliminary test. The pulsewidth at which the knee comes out of low-level saturation (rest position) is taken as $u=0$ and the pulsewidth at which the knee goes into high-level saturation (extension) corresponds to $u=1$. Finally, an estimation of the system's equilibrium curve for normalized pulsewidth and angle is obtained from the measured data which will be useful to prepare the input pattern for controller design.

After this first stage, the data set for control design is generated. A carefully designed input signal is applied to the electrically stimulated muscle. The pulsewidth signal used has specific stochastic characteristics. The goal is to generate a pulsewidth input sequence which leads to an almost uniform distribution of the angle spanning almost all the achievable range of knee joint motion. The normalized pulsewidth signal changes at each sampling time to a new value with a predetermined switching probability. When the pulsewidth value has to be changed, the new value is drawn from a uniform distribution over the interval $[0-1]$ and is processed by the inverse static (equilibrium) curve of the knee joint dynamics, estimated in the preliminary test. An example of I/O data used for controller design is shown in Fig. 4.

Finally, the last step of the experimental session consists in testing the controller designed according to the method described in Section IIIwith the data collected at the previous stage. Examples of theses results will be presented in Section IV.

\section{Controller Design Using VirTual Reference FEEDBACK TUNING}

Using the data collected at the beginning of the experimental session, the VRFT allows the design of a controller for an un-
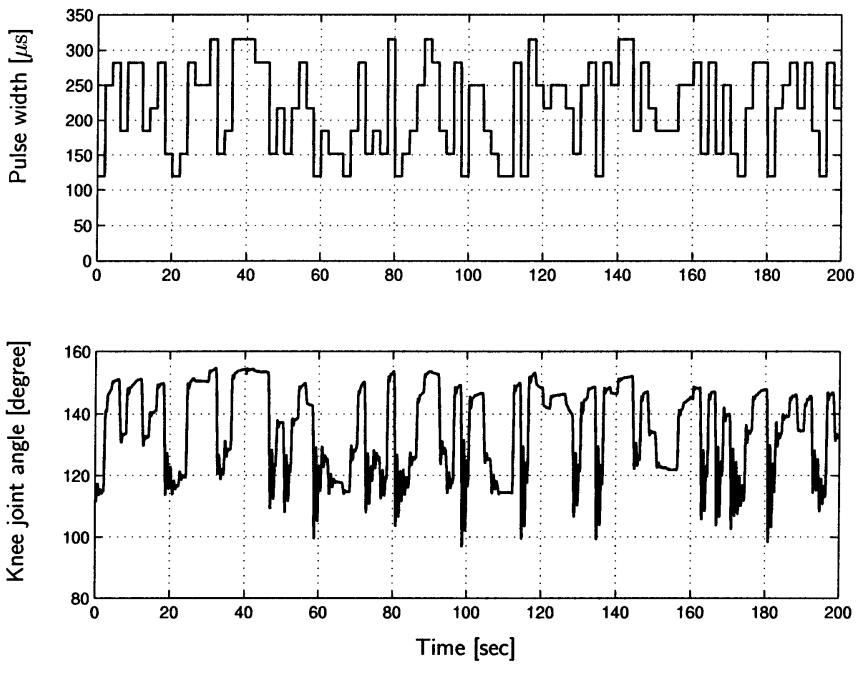

Fig. 4. Input/output data collected on a paraplegic. This is the data set used for the design of a linear controller according to the VRFT strategy. The upper section represents the stimulation input; the lower part is the measured knee joint angle.

known system without resorting to the identification of a model of the system.

The VRFT method approximately solves a model-reference problem. This means that the control specifications are assigned via a reference model which describes the desired behavior of the closed-loop system [17]. This reference model, given by a pulse transfer function $M(q)$, is used to define in a simple and effective way the basic characteristics of the control system such as its settling time, the allowed overshoot, etc. In standard applications, a second order model is usually satisfactory.

In the VRFT approach, a following closed-loop system is considered:

$$
\left\{\begin{array}{l}
y(t)=P(q) u(t) \\
u(t)=C(q ; \theta)(r(t)-y(t))
\end{array}\right.
$$

Here, the single-input-single-output (SISO) linear plant is described by the pulse transfer function $P(q)$ where $q$ is the forward shift operator $(x(t+1)=q x(t))$. Further in (1), $C(q ; \theta)$ is a linear one-degree-of-freedom controller belonging to a given family of linear pulse transfer function controllers $\{C(q ; \theta)\}_{\theta \in \mathbb{R}^{n}}$ parameterised by the $n$-dimensional real vector $\theta$. The signals $u(t)$ and $y(t)$ are respectively the input and the output of the considered plant. The signal $r(t)$ is the reference signal of the control loop.

Let us assume that a set of I/O data $\{u(t), y(t)\}_{t=1, \ldots, N}$ has been collected from an experiment on the plant and that a reference model $M(q)$ has been chosen. The goal is to solve a model reference problem, i.e., to find

$$
\bar{\theta}=\arg \min _{\theta} J_{M R}(\theta)
$$

where

$$
J_{M R}(\theta)=\left\|\frac{P(z) C(z ; \theta)}{1+P(z) C(z ; \theta)}-M(z)\right\|_{2}^{2} .
$$

To this aim, given the measured $y(t)$ (i.e., the actual signal measured at the output of the plant), consider a reference $r_{v}(t)$ 
such that $M(q) r_{v}(t)=y(t)$. Such a reference is called "virtual" because it does not exist in reality and in fact it was not used in the generation of $y(t)$. So, in this framework, $y(t)$ is the desired output of the closed-loop system when the reference signal is $r_{v}(t)$. Finally, compute the corresponding tracking error $e(t)=$ $r_{v}(t)-y(t)$.

Notice that, even though the plant transfer function $P(q)$ is not known, when the plant is fed by $u(t)$ (the actually measured input signal), it generates $y(t)$ (the corresponding measured output signal). Therefore, a good controller generates $u(t)$ when fed by $e(t)$, at least in the condition when the reference signal is the virtual reference $r_{v}(t)$. The idea is then to search for such a controller. Since both signals $u(t)$ and $e(t)$ are known, this task reduces to the identification problem of describing the dynamical relationship between $e(t)$ and $u(t)$ by using the family of linear models $\{C(q ; \theta)\}_{\theta \in \mathbb{R}^{n}}$.

In the following, the algorithm implementing the above idea will be briefly outlined. In the algorithm, the identification of the controller is addressed by minimizing the classical least-squares identification criterion (see [28]).

Design Algorithm: Given the reference model $M(q)$, the family of controllers $\{C(q ; \theta)\}_{\theta \in \mathbb{R}^{n}}$ and the set of data $\{u(t), y(t)\}_{t=1, . ., N}$, do the following.

1) Calculate:

- a virtual reference $r_{v}(t)$ such that $y(t)=$ $M(q) r_{v}(t)$, and

- the corresponding tracking error $e(t)=r_{v}(t)$ $y(t)$.

2) Filter the signals $e(t)$ and $u(t)$ with a suitable filter $L(q)$, obtaining $e_{L}(t)$ and $u_{L}(t)$ :

$$
e_{L}(t)=L(q) e(t), \quad u_{L}(t)=L(q) u(t) .
$$

3) Estimate the controller parameter vector

$$
\hat{\theta}_{N}=\arg \min _{\theta} J_{V R}^{N}(\theta)
$$

where

$$
J_{V R}^{N}(\theta)=\frac{1}{N} \sum_{t=1}^{N}\left(u_{L}(t)-C(q ; \theta) e_{L}(t)\right)^{2} .
$$

Notice that (5) is quadratic in the parameter vector $\theta$ and all the computations are directly performed on the measurement data, assumed that the filter $L(z)$ is given.

The filter used in the experiments is

$$
L(z)=\frac{M(z)(1-M(z))}{U(z)}
$$

where $U(z)$ is a model of the input signal such that $u(t)=$ $U(q) \nu(t)$ where $\nu(t)$ is a white noise with unit variance, so that the power density spectrum of the input can be modeled as $\phi_{u}(\omega)=\left|U\left(e^{j \omega}\right)\right|^{2}$. More information about this problem can be found in [22], [23].

In this work, we consider the class of PID controllers as having the following form:

$$
C(q ; \theta)=K_{p}+K_{i} \frac{T_{s}}{1-q^{-1}}+K_{d} \frac{1-q^{-1}}{T_{s}}
$$

where $T_{s}$ is the sampling time of the system. Notice that since the controller is linear in the parameters $\theta=\left[K_{p} K_{i} K_{d}\right]$ the performance index (5) is quadratic and the parameter estimate $\hat{\theta}_{N}$ can be easily found.

The practical implementation of the controller has been realized with a standard implementation of an antiwindup scheme [29]. This avoids undesirable effects due to the interaction of the integral action of the controller and the control action saturation given by the limited pulsewidth values that can be delivered to the muscle.

The proposed algorithm can be modified in order to obtain nonlinear controllers. The use of nonlinear controllers is strongly advisable in FES control. In fact, it has been evidenced by experiments that the muscle-joint system is not a linear plant. The main sources of nonlinearity are gravity and muscle contraction dynamics. So, a nonlinear version of VRFT has been designed, where the control action is given by

$$
u(t)=\sum_{i=1}^{S} g_{i}(v(t-i+1))
$$

where

$$
v(t)=\frac{1}{1-q^{-1}} e(t)
$$

and

$$
g_{i}(v(t-i+1))=\sum_{k=1}^{M} \theta_{k} e^{-\left(r_{i}\left(v(t-i+1)-c_{k}\right)\right)^{2}}
$$

with $r_{i} \in \mathbb{R}$ for all $i=1, \ldots, S$ and $\theta_{k}, c_{k} \in \mathbb{R}$ for all $k=$ $1, \ldots, M$.

The previous representation corresponds to the use of $S$ nonlinear functions parameterised by radial basis functions networks (RBFNs). Each RBF network is built using $M$ radial basis functions. The parameters $c_{k}$ and $r_{i}$ are chosen a priori: they are respectively the centres and the widths of the basis functions. So, the control action is linear in the parameters $\theta_{k}$ which could be estimated by using the same algorithm described before.

In this work, $S=4$ and $M=6$ have been chosen. So, the control action of (8) can be represented by using the scheme of Fig. 5.

\section{EXPERIMENTAL RESULTS}

First, the controller parameters are computed using the I/O data, collected according to the protocol of Section II, and the following reference model:

$$
M(q)=\frac{0.016 q^{-6}}{1-1.75 q^{-1}+0.765 q^{-2}} .
$$

This model has rise time $T_{r}=1 \mathrm{~s}$ and damping $D=0.99$.

Once that a controller has been designed for a subject following the algorithm of Section III, two kinds of tests were performed in order to qualitatively evaluate the properties of the design: a tracking tests and a disturbance rejection test.

In a tracking test stair-like or square-wave reference signals are used. When performing such tests using linear design, the 


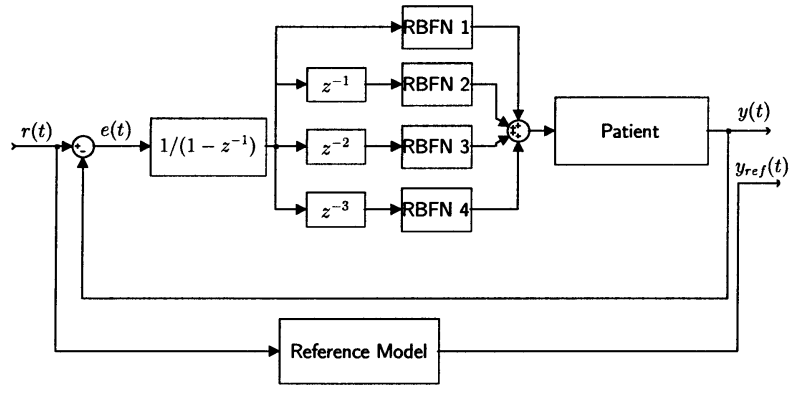

Fig. 5. Nonlinear VRFT control scheme.
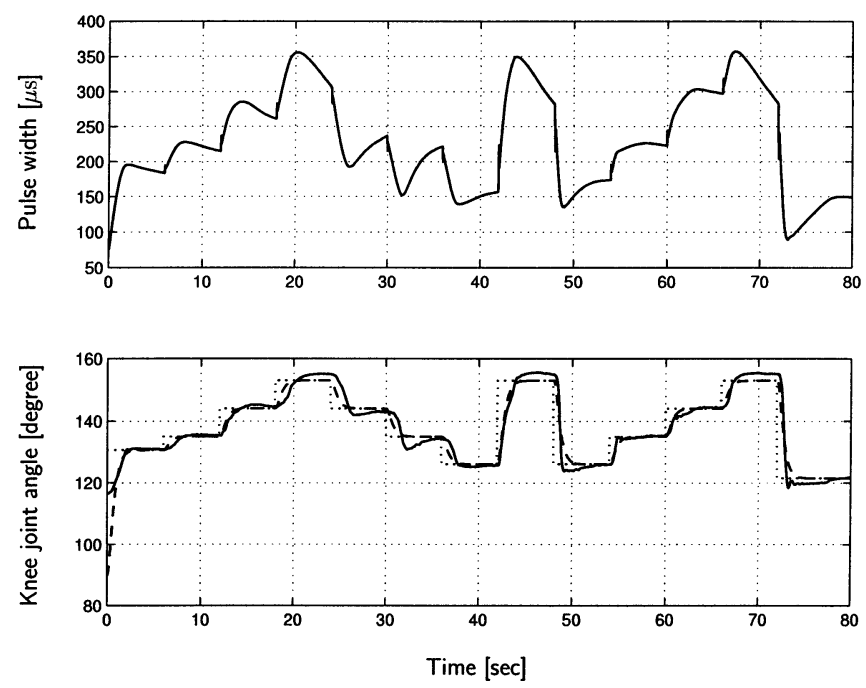

Fig. 6. Tracking of a sequence of steps using a linear PID controller applied to a healthy subject. The upper part represents the control action (stimulation input). The lower section is the knee joint angle (-measured angle, ....reference, -reference filtered by the tracking reference model).

reference signal is usually not spanning all the extension range of the knee joint and it is limited to about $120^{\circ}-150^{\circ}$ in healthy subjects and $130^{\circ}-140^{\circ}$ in paraplegics who have smaller extension ranges. Within these ranges, the system behavior is considered to be relatively linear.

The disturbance rejection test consists in pushing or pulling the shank (or putting a known weight on the ankle) while the controller has to maintain a fixed reference angle. Observing how fast and smoothly the controller leads the leg back to the reference position, one can obtain an impression of the degree of robustness of the closed-loop system.

In the following, first, results of the linear control for the healthy subject will be presented. Then, results of linear and nonlinear control for the paraplegic subject will be shown.

\section{A. Healthy Subject}

In Fig. 6, an example of a tracking test is shown for the linear controller (7). Here, a stair-like reference signal was used. The reference range has been kept limited to the central region (about $120^{\circ}-150^{\circ}$ ) of the extension range of the healthy knee, so that the linear controller could be effective.

Healthy subjects have of course the ability to perform voluntary muscle contractions. Although the subject was blindfolded during the experiment and could not see the reference trajectory
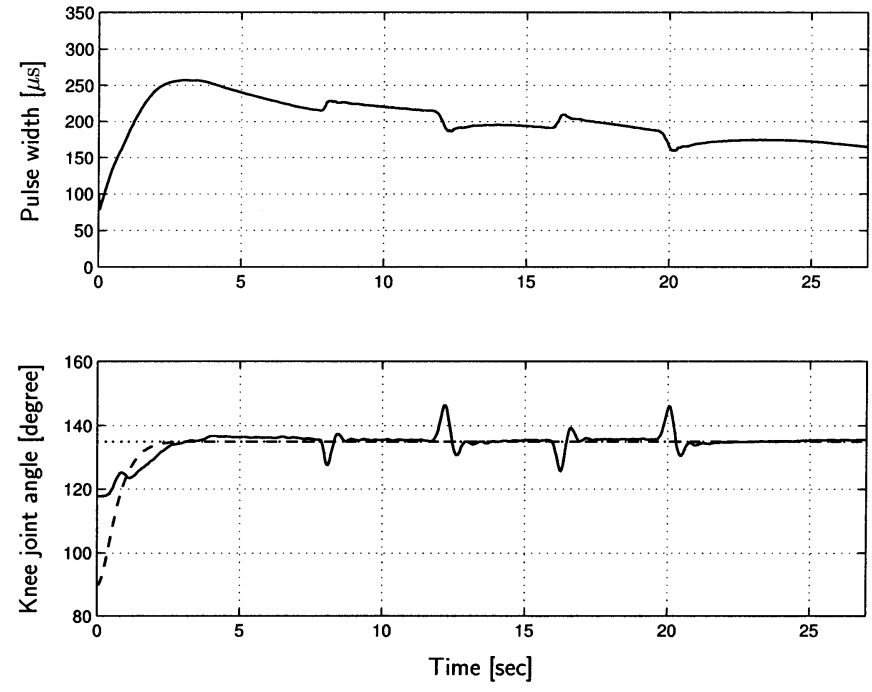

Fig. 7. Verification of the PID controller's disturbance rejection property on a healthy subject. The upper part represents the control action (stimulation input). The lower section is the knee joint angle (- measured angle, ... reference, -reference filtered by the tracking reference model). At about $t=8 \mathrm{~s}$ and $t=17 \mathrm{~s}$ the subject leg has been pushed down. At about $t=12 \mathrm{~s}$ and $t=20 \mathrm{~s}$ the subject leg has been lifted up.

on the laptop screen, significant interaction of feedback controller and subject could be observed. In fact, the healthy subject had the tendency to induce normal muscle contractions by himself which were initially triggered and generated by FES when the reference changed to a higher knee extension level. As a result, the controller used a reduced intensity of the control action to track the reference. In Fig. 6, the pulsewidth is continously declining after a higher knee joint extension level is reached; this indicates that increasing voluntary torque is present.

When the reference changed to a lower level the subject needed some time to relax the muscles, which can be seen in the slowly raising pulsewidth after the lower level has been reached; voluntary muscle contraction is decreasing during this period.

However, the closed-loop system shows good tracking properties. Small tracking offsets result from voluntary muscle contractions. The knee joint angle accurately follows the reference angle while the control signal is varying smoothly.

From Fig. 7, disturbance rejection properties of the linear controller can be evaluated. When the leg is pushed or pulled by the experimenter, the controller is able to effectively lead the leg to the original position. In this specific case, the leg has been gently pushed down at about $t=8 \mathrm{~s}$, while at about $t=17 \mathrm{~s}$ some more energy has been put in pushing it down. At about $t=12 \mathrm{~s}$ and $t=20 \mathrm{~s}$ the subject leg has been pulled up. The role played by the healthy subject who interacts with the controller is also evident from this plot. As result, the controller is enabled to use lower intensities in the control action.

\section{B. Paraplegic Subject}

A paraplegic subject does not have the ability of contracting muscles, so he cannot interact with the controller. As expected, no significant interaction of subject and controller could be detected. 

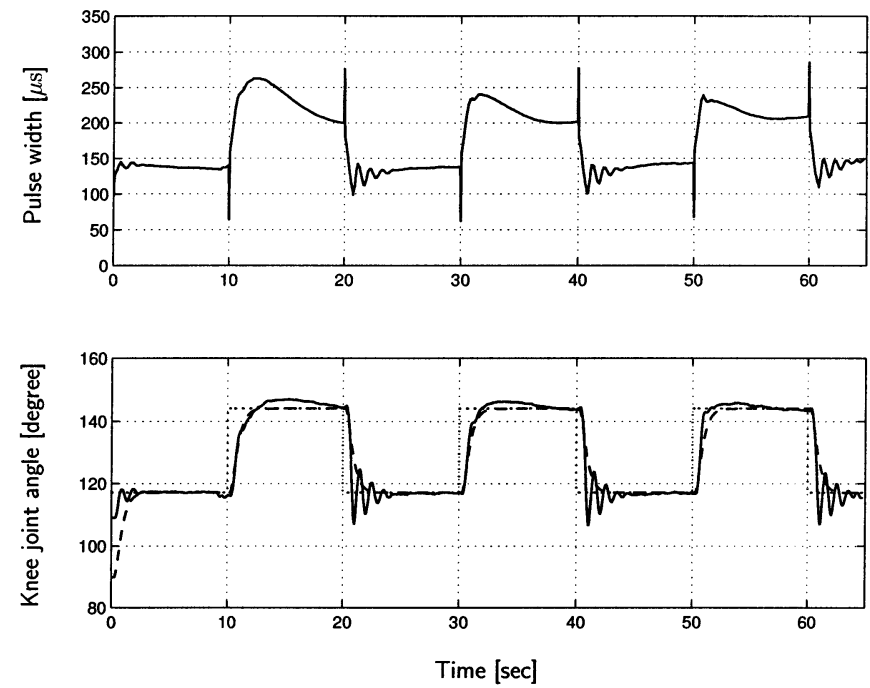

Fig. 8. Tracking of a square wave using a linear PID controller applied to a paraplegic subject. The upper part shows the control action (stimulation input). The lower section represents the knee joint angle (-measured angle,- - reference filtered by the tracking reference model).

Fig. 8 represents a tracking test where the square-wave reference signal spans almost the full extension range of the knee (about $110^{\circ}-150^{\circ}$ ). This test clearly shows that a linear controller was not completely effective when applied to this large joint angle range. It is evident that the linear controller shows undesirable oscillations. The spikes in the control action are an effect of the derivative action in the PID controller. The controller notices a step variation in the error signal and the derivative action generate a pulse signal component in the control action. This can be avoided by implementation of the PID controller with output derivation instead of error derivation.

After the linear controller test, a nonlinear controller was evaluated using the same reference trajectory shown in Fig. 8. The outcome of this tracking test is depicted in Fig. 10.

To evalutate the tracking performance of linear and nonlinear control, the root-mean-square (rms) error given by

$$
\mathrm{rms}=\sqrt{\frac{\sum_{t=1}^{N_{c}}(y(t)-M(q) r(t))^{2}}{N_{c}}}
$$

was calculated. Here, $N_{c}$ is the number of samples of the control test. For the linear controller the rms error was $3.82^{\circ}$, corresponding to $9.25 \%$ of the knee angle range covered. For the nonlinear controller the rms error was $3.54^{\circ}$, corresponding to $7.73 \%$ of the knee angle range.

As shown by Figs. 8 and 10 the use of a nonlinear controller improves the tracking quality by elimination of undesired oscillations and by use of less control energy.

From Fig. 9, disturbance rejection properties of the linear controller can be evaluated.

From Fig. 9, disturbance rejection properties of the linear controller can be evaluated. In this specific case, the leg has been gently pushed down at about $t=8 \mathrm{~s}$ and $t=12 \mathrm{~s}$. At about $t=16 \mathrm{~s}$ and $t=21 \mathrm{~s}$ the subject leg has been pulled up. When the disturbances occur, the knee joint angle initially drops or increases depending on the kind of disturbance but the
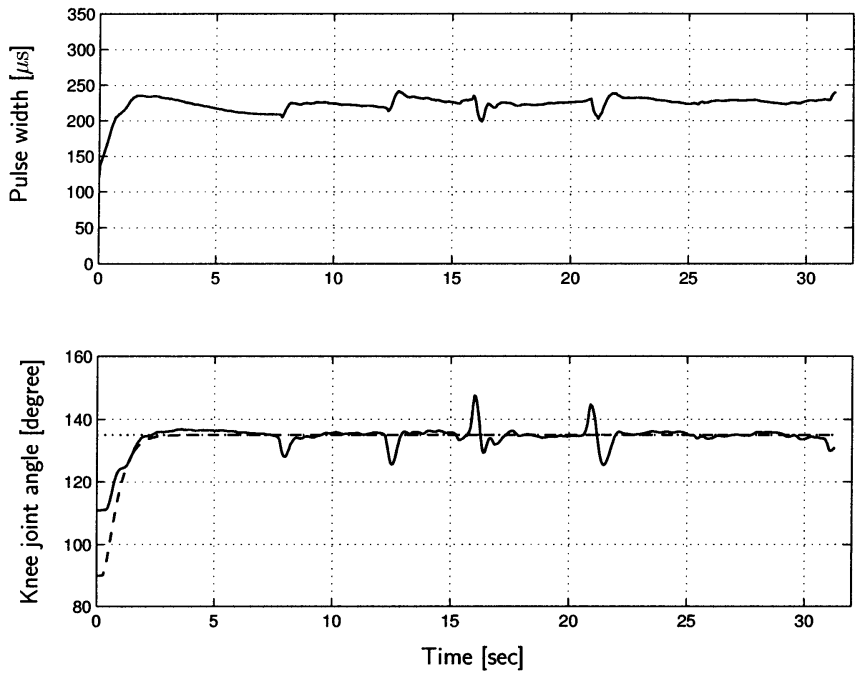

Fig. 9. Verification of the PID controller's disturbance rejection properties on a paraplegic subject. The upper section represents the control action (stimulation input). The lower part is the knee joint angle (- measured angle, - reference filtered by the tracking reference model). At about $t=8 \mathrm{~s}$ and $t=12 \mathrm{~s}$ the subject leg has been pushed down. At about $t=16 \mathrm{~s}$ and $t=21 \mathrm{~s}$ the subject leg has been lifted up.
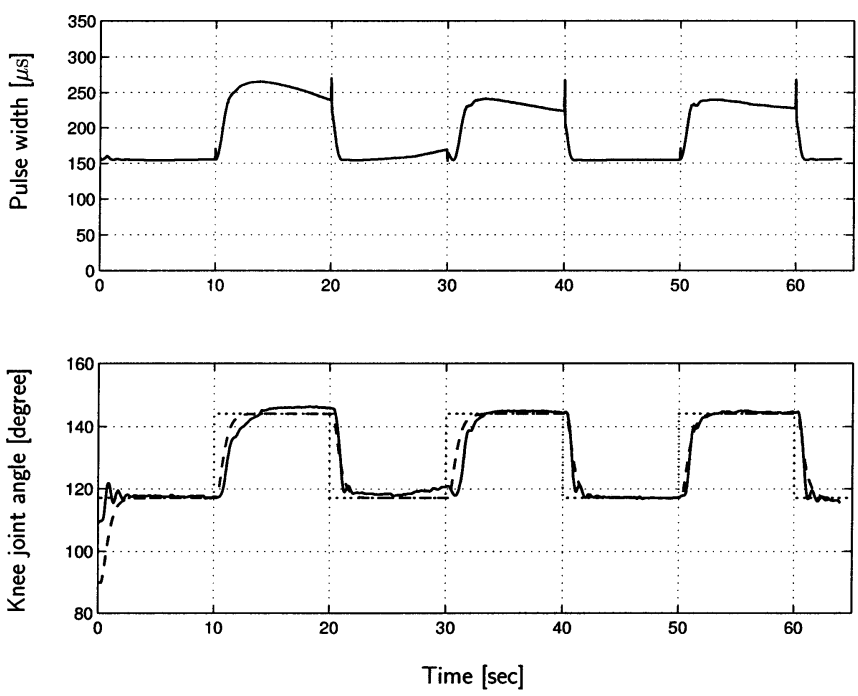

Fig. 10. Tracking test of a nonlinear controller on a paraplegic subject. The upper section represents the control action (stimulation input). The lower part is the knee joint angle (- measured angle,-reference filtered by the tracking reference model).

controller automatically brings the shank back to the position to be maintained. Notice that in this case no interaction between subject and controller is evidenced.

\section{Discussion AND CONCLUSION}

In this brief paper, a controller designed directly from data applied to FES control of the knee joint movement has been developed and successfully tested in experiments with intact and paraplegic subjects. Based on a single measured I/O data set, the design is yielding a linear feedback controller with good tracking and disturbance rejection properties for limited joint angle ranges. For larger ranges the tracking performance of the linear controller is suffering from insufficient compensation of 
nonlinear effects, mainly due to gravity. However, for these situations, the introduction of a nonlinear static mapping in the controller considerably improves the tracking performances.

The Virtual Reference Feedback Tuning strategy has been of great help since it has considerably shortened the experimental session time. In fact, when developing a model based "controlled-centered" neuroprosthesis, the following steps are usually performed (see [1], [6], [10], [12]-[16]).

1) Measurement of the recruitment curve, to determine threshold and saturation pulsewidth for the quadriceps.

2) Open-loop identification tests: measurements of $\mathrm{I} / \mathrm{O}$ data sets for different stimulation levels are performed on the patient and the results are arranged into estimation and validation sets.

3) Modeling the relation between stimulation pulsewidth and angle: model parameters and structure are estimated and validation tests are performed on the patient.

4) Controller design: a controller is designed according to a given strategy and based on the model estimated at the previous step. Finally, the controller is applied to the patient for final test and validation.

In particular, we experienced that step 3 could last up to 20 min and we noticed that sometimes the muscle contraction properties could slightly change. The use of a direct method (not model based), like VRFT, that avoid the identification step 3 is helpful in reducing the duration of a session.

A second major problem in FES control is the increasing fatigue of the stimulated muscles. Fatigue effects can be very different from subject to subject and from day to day. Basically, they are evident in two ways: 1) during a single stimulation session, after a while, the muscle is not able to contract effectively; i2) if many subsequent stimulation sessions are repeated, the effect described in 1) becomes evident earlier. This second evidence of fatigue is considerably reduced using VRFT. In fact, the absence of a modeling and/or parameter identification step in the experimental protocol considerably reduces the time for control design (model design, parameter estimation, model validation, controller tuning, etc.). The other effect of fatigue is visible during a single session: muscle fatigue causes a shift in the equilibrium curve of the system, which describes the static relation between pulsewidth and knee angle. The curve will be shifted to higher pulsewidth regions; so, if the muscle fatigues, the pulsewidth must be increased to maintain a given angle. In this work, this effect is successfully compensated by the integral action in the controller and the gain of the controller does not need to be adjusted. In fact, since integral action is embedded into the controller, the controlled neuroprosthesis can successfully counteract slowly varying system properties due to muscle fatigue or any other constant disturbance.

\section{REFERENCES}

[1] F. Previdi, M. Ferrarin, S. M. Savaresi, and S. Bittanti, "Closed loop control of FES supported standing up and sitting down using virtual reference feedback tuning: A simulation study," in Proc. IFAC Symp. Modeling and Control in Biomedical Systems, Melbourne, Australia, Aug. 2003.

[2] K. J. Hunt, M. Munih, N. Donaldson, and F. M. D. Barr, "Optimal control of ankle joint moment: Toward unsupported standing in paraplegia," IEEE Trans. Automat. Contr., vol. 43, p. 819, June 1998.
[3] T. Schauer, K. J. Hunt, A. Ronchi, M. H. Fraser, and W. Stewart, "Robust control of knee-joint motion," in Proc. 6th Annu. Conf. Int. Functional Electrical Stimulation Society, Cleveland, OH, June 2001.

[4] N. Donaldson and C.-H. Yu, "FES standing: Control by handle reactions of leg stimulation (CHRELMS)," IEEE Trans. Rehab. Eng., vol. 4, pp. 280-284, Dec. 1996.

[5] R. Riener and T. Fuhr, "Patient-driven control of FES-supported standing up: A simulation study," IEEE Trans. Rehab. Eng., vol. 6, pp. 113-124, June 1998.

[6] F. Previdi and E. Carpanzano, "Design of a gain scheduling controller for knee-joint angle control by using functional electrical stimulation," IEEE Trans. Contr. Syst. Technol., vol. 11, pp. 310-324, May 2003.

[7] R. Riener and J. Quintern, "A physiologically based model of muscle activation verified by electrical stimulation," Bioelectrochem. Bioenerget., vol. 43, pp. 257-264, 1997.

[8] G.-C. Chang, J.-J. Luh, G.-D. Liao, J.-S. Lai, C.-H. Cheng, B.-L. Kuo, and T.-S. Kuo, "A neuro-control system for the knee joint position control with quadriceps stimulation," IEEE Trans. Rehab. Eng., vol. 5, pp. 2-11, Mar. 1997

[9] M. Ferrarin and A. Pedotti, "The relationship between electrical stimulus and joint torque: A dynamic model," IEEE Trans. Rehab. Eng., vol. 8 , pp. 342-352, Sept. 2000.

[10] M. Ferrarin, F. Palazzo, R. Riener, and J. Quintern, "Model-based control of fes-induced single joint movements," IEEE Trans. Neural Syst. Rehab. Eng., vol. 9, pp. 245-257, Sept. 2001.

[11] R. Riener, "Model-based development of neuroprostheses for paraplegic patients," Phil. Trans. Royal Soc. London Ser. B-Biol. Sci., vol. 354, pp. 877-894, May 1999.

[12] T. Schauer and K. Hunt, "Linear modeling and controller design for the single limb movement of paraplegics using FES," in Proc. 4th IFAC Symp. Modeling and Control in Biomedical Systems, Greifswald, Germany, Mar.-Apr. 2000, pp. 7-12.

[13] F. Previdi, "Identification of black-box nonlinear models for lower limb movement control using functional electrical stimulation," Contr. Eng. Pract., vol. 10, no. 1, pp. 91-99, June 2002.

[14] F. Previdi and M. Lovera, "Identification of a class of nonlinearly timevarying models," Int. J. Adapt. Contr. Signal Processing, vol. 17, pp. 33-50, 2003.

[15] T. Schauer and K. J. Hunt, "Nonlinear predictive control of knee-joint angle using FES," in Proc. IFESS Conf. 2000, Aalborg, Denmark, June 2000.

[16] T. Schauer, M. H. Fraser, W. Stewart, K. J. Hunt, and F. Previdi, "Identification of a biomechanical system using neural networks," in Proc. IFAC Workshop .Adaptation and Learning in Control and Signal Processing, Como, Italy, Aug. 2001, pp. 49-56.

[17] H. Nijmeijer and S. M. Savaresi, "On approximate model-reference control of siso discrete-time nonlinear systems," Automatica, vol. 34, pp. 1261-1266, 1998

[18] S. M. Savaresi and G. O. Guardabassi, "Approximate feedback linearization of discrete time nonlinear systems using virtual input direct design," Syst. Contr. Lett., vol. 32, pp. 63-74, 1997.

[19] - "Approximate I/O feedback linearization of discrete time nonlinear systems via virtual input direct design," Automatica, vol. 34, pp. 715-722, 1998.

[20] G. O. Guardabassi and S. M. Savaresi, "Virtual reference direct design method: An off-line approach todata-based control system design," IEEE Trans. Automat. Contr., vol. 45, pp. 954-959, 2000.

[21] — , "Approximate linearization via feedback—an overview," Automatica, vol. 27, pp. 1-15, 2001

[22] M. C. Campi, A. Lecchini, and S. M. Savaresi, "Virtual reference feedback tuning: A direct method for thedesign of feedback controllers," Automatica, vol. 38, pp. 1337-1346, 2002.

[23] —_, "Virtual reference feedback tuning for two degree of freedomcontrollers," Int. J. Adapt. Contr. Signal Processing, vol. 16, pp. 355-371, 2002.

[24] — "An application of the virtual reference feedback tuning (VRFT) method to a benchmark active suspension system," Eur. J. Contr., vol. 9 , pp. 66-76, 2003.

[25] F. Previdi, T. Schauer, A. Lecchini, S. M. Savaresi, and K. J. Hunt, "Virtual reference feedback tuning forknee joint position control using functional electrical stimulation," in Proc. Eur. Control Conf., Porto, Portugal, Aug.-Sept. 2001.

[26] Measuring System for 3D-Motion Analysis. Isny im Allgäu, Germany: Zebris Medizintechnik GmbH, 1999.

[27] User's Manual, Real Time Toolbox, Version 3.0. Prague, Czech Republic: Humusoft S.R.O, 1999.

[28] L. Ljung, System Identification: Theory for the User. Upper Saddle River, NJ: Prentice-Hall, 1999.

[29] K. J. Åström and B. Wittenmark, Computer-Controlled Systems: Theory And Design. Upper Saddle River, NJ: Prentice-Hall, 1997. 